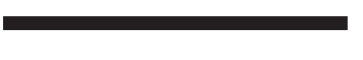

AUTORES:

Sebastián Feu ${ }^{1}$

Sergio J. Ibáñez

Javier García-Rubio

Antonio Antúnez Medina

'Universidad de Extremadur

${ }^{2}$ Universidad Autónoma de Chile

https://doi.org/10.5628/rpcd.17.S1A.50

\section{La investigación sobre \\ la enseñanza de los deportes de invasión y su transferencia al contexto escolar}

\section{PALABRAS CLAVE:}

Deportes de Invasión. Modelos de enseñanza.

Investigación. Transferencia. Escuela.

\title{
RESUMEN
}

El objetivo de este trabajo es analizar las dificultades para generalizar los resultados de la investigación y transferir las ventajas que aportan los modelos de enseñanza a la escuela. La comparación entre estudios es compleja y dificulta una transferencia clara a los docentes, estas dificultades se centran en: i) existe un predominio de investigaciones parciales y con diferentes variables, ii) los diseños de investigación en ocasiones son muy diferentes, iii) las características del deporte escolar y extraescolar difieren en objetivos, heterogeneidad de la población y temporalizaciones, iv) el tamaño muestral es pequeño y no se controla el tamaño del efecto, v) se desconoce el contenido y la validez de los programas empleados en las investigaciones, vi) se desconoce cómo es el control del proceso de comunicación docente - aprendiz, vii) en los estudios iniciales no hay indicadores de la eficacia en el juego que permitan la comparación de resultados. Es necesario realizar investigaciones desde una perspectiva holística e integral, teniendo en cuenta la realidad de cada contexto formativo, los aprendices y docentes.

Research on the teaching of invasion sports

\section{ABSTRACT}

The aim of this work is to analyze drawbacks to generalize research findings in this topic, and to transfer the advantages of teaching models on the school. The applicability of the results has been difficult due to the complexity of the comparison between studies, mainly because of: i) researches have different variables; ii) research design are different; iii) sport during the scholarship have different aims, sample and planning inside and outside the school; iv) samples are small and effects sizes are not controlled; v) contents and validity of the sports programs are unknown; vi) interactions teacher-student are unidentified; vii) first studies lack of efficacy indicators that allow results comparisons. An integral and holistic research approach is necessary, keeping in mind the specific context, learners and teachers.

KEY-WORDS:

Invasion sports. Teaching models.

Research. Transfer. School. 
Para la enseñanza de los deportes de invasión en la edad escolar se han contrastado diversos modelos de enseñanza - aprendizaje: el tradicional, la enseñanza basada en la comprensión del juego y los modelos que surgen de la pedagogía no lineal.

Algunos docentes consideraban que en la enseñanza de deporte debe primar el desarrollo de habilidades antes que la comprensión del juego ${ }^{(31,33)}$.Los modelos tradicionales centran su atención en las habilidades técnicas para después aplicarlas en e

l entorno real, produciéndose una disociación entre aprendizaje motriz, la técnica y la cognición, los aspectos tácticos ${ }^{(18)}$. Estos modelos han predominado en el ámbito escolar. Los deportes de invasión el juego se desarrollan de forma dinámica y no lineal entre los practicantes y el contexto de juego ${ }^{(3)}$. En la literatura científica y académica se ha tratado de dar respuesta a la enseñanza de los deportes de invasión enfrentando al aprendiz a los problemas cambiantes del juego. Como reacción a los modelos tradicionales surgen los modelos de aprendizaje con una aproximación hacia la comprensión del juego (Game Centred Approaches, GCA) a través de la propuesta de ${ }^{(4)}$ de los Teaching Game for Understanding - TGFU. Este modelo surge como una herramienta operativa, con un enfoque práctico, para diseñar tareas que centran la atención en el alumno, el cual debe buscar soluciones que le llevarán a comprender los aspectos tácticos del juego. A lo largo del mundo han surgido diferentes propuestas con principios similares, Games Sense ${ }^{(8,9)}$, Tactical Games ${ }^{(24}$ 14), Sport Education Model (32), Play Practice ${ }^{(19)}$, Concept Based Games ${ }^{(21)}$. Algunos autores han considerado idóneos estos modelos para aplicarse en la escuela por aproximarse a las teorías del constructivismo (1).

Otro enfoque para afrontar el aprendizaje del deporte surge de la interacción de la psicología ecológica y la teoría de los sistemas dinámicos, la pedagogía no lineal ${ }^{(6)}$. Ante los problemas cambiantes que se producen en el juego, el aprendizaje se debe producir desde una perspectiva ecológica donde los aprendices son los que deben resolver un problema interaccionando con los condicionantes cambiantes de la tarea que forman parte del juego ${ }^{(4,30)}$. Los principios del aprendizaje de los TGFU, muestreo, la complejidad táctica, exageración y representación, podrían encontrar un soporte teórico en la pedagogía no lineal (35)

Fundamentándose ya en la pedagogía no-lineal se plantea el aprendizaje del deporte a partir de poner en un primer plano la relación entre los aprendices y el contexto a través de los condicionantes de la tarea (Contraints-Led Approach, CLA) ${ }^{(6)}$. La alteración de los condicionantes de la tarea favorece que surjan una amplia variedad de patrones de comportamiento funcionales y autosuficientes dentro del contexto específico del juego deportivo ${ }^{(6)}$. Los condicionantes de la tarea favorecen la aparición de nuevos retos a los que debe adaptarse el aprendiz.

Siguiendo cualquiera de los modelos de aprendizaje del deporte se han realizado investigaciones para contrastar su eficacia en función de diversos criterios, encontrándose dificultades a la hora de comparar los diferentes estudios ${ }^{(33,23)}$ para obtener conclusiones poran de forma más amplia los modelos que se fundamentan en la pedagogía no lineal? El objetivo de este trabajo es analizar las dificultades para generalizar los resultados de la investigación y transferir las ventajas que aportan los modelos de enseñanza centrados en la comprensión del juego y/o en la pedagogía no lineal en su aplicación en la escuela.

\section{CONDICIONANTES PARA LA TRANSFERENCIA \\ DE LAS EVIDENCIAS OBTENIDAS \\ POR LA INVESTIGACIÓN A LA ESCUELA}

Revisiones sistemáticas de la literatura ponen de manifiesto discrepancias entre las ventajas de un modelo u otro ${ }^{(33,23)}$, observándose también algunas discrepancias entre los modelos teóricos y las evidencias científicas ${ }^{(23)}$. A pesar de la gran cantidad de investigaciones sobre los modelos para la enseñanza de los deportes de equipo la transferencia al ámbito escolar ve condicionada por la dificultad para extrapolar el deporte extraescolar al escolar, por algunas dificultades en el diseño de las investigaciones y la falta de materiales adecuados para los docentes. Los condicionantes para transferir estas evidencias son:

I) PREDOMINTO DE ESTUDTOS PARCIALES EN LA INVESTIGACIÓN EN LOS GCA.

La investigación sobre los beneficios de los GCA se han realizado en numerosas ocasiones teniendo en cuenta variables dependientes aisladas: el aprendizaje de las habilidades técnica, el conocimiento declarativo y/o procedimental, la toma de decisiones, el conocimiento del juego, o variables socio afectivas ${ }^{(23,16)}$, dificultando el conocimiento del alcance de las investigaciones en los escolares. Son más interesantes las conclusiones obtenidas en estudios que tienen en cuenta una perspectiva más integral y holística del aprendizaje ${ }^{(25,11)}$, pues podemos conocer los efectos de un programa sobre diversas áreas de su desarrollo de los aprendices.

II) DISEÑOS DE LA INVESTIGACIÓN EN EL ESTUDIO DE LOS GCA.

La investigaciones para conocer los efectos de los modelos de aprendizaje del deporte ha realizado desde diversos enfoques de investigación ${ }^{(18)}$, con estudios cuantitativos ${ }^{(33)}$ con diseños cuasi-experiementales y estudios cualitativos con un enfoques naturalistas ${ }^{(33,17)}$. Dentro de los estudios cuasi-experiementales hay diversidad de diseños, algunos con grupo control y otros sin grupo control, dificultando todo ello la comparación de los resultados de la investigación ${ }^{(33,23)}$. La combinación de diversos enfoques de investigación, cualitativo y cuantitativo, van a permitir tener un conocimiento más aproximado a la realidad de la aplicación de los GCA en el aula, siempre que se realicen centrados tanto en el aprendiz como en el docente. 
III) LAS POBLACIONES ESTUDIADAS.

Los estudios sobre las modelos de enseñanza del deporte se han realizado en diferentes contextos de investigación, deporte escolar ${ }^{(11)}$ y extraescolar ${ }^{(10)}$, contextos muy diferentes. La educación física viene regulada por el currículum, en donde los deportes de invasión son un contenido más. El proceso de enseñanza-aprendizaje se vertebra en unidades didácticas que habitualmente oscilan entre 10 y 15 sesiones en el mejor de los casos, donde el objetivo es comprender el juego deportivo y su papel dentro de la cultura, así como servir de vehículo para el desarrollo de habilidades perceptivas, decisionales y motrices del alumno. Es necesario plantearse una adecuada selección de contenidos ajustados a tiempo disponible con un numero de tareas razonables para su desarrollo. La modificación de los condicionantes de la tarea (reglas, espacio, tiempo, jugadores,...) debe servir para ajustar la progresión de los problemas de aprendizaje ${ }^{(6,7)}$. Por otro lado, las habilidades previas en el deporte son más heterogéneas entre los practicantes del ámbito escolar que en el extraescolar y esto debe ser tenido en cuenta en los diseños de investigación sobre todo cuando hay grupos de control y se comparan modelos de aprendizaje.

IV) LA SELECCIÓN DE LA MUESTRA Y GENERALIZACIÓN DE LOS RESULTADOS.

Los trabajos de investigación sobre la enseñanza comprensiva de los deportes de invasión se han realizado en diferentes deportes diferentes y con diferentes tamaños de la muestra. En la escuela los grupos son naturales y es complicado realizar una aleatorización de los participantes. Esto dificulta la generalización de los resultados obtenidos a través los análisis estadísticos, ya que el uso de muestras reducidas en los diseños cuasi-experimentales en muchos casos no han ido acompañado del cálculo del tamaño del efecto para conocer la relevancia de la significación obtenida, medida que en la actualidad se va incorporando en algunos trabajos ${ }^{(11,27)}$.

V) LA VALIDEZ Y DIFUSIÓN DE LOS PROGRAMAS BASADOS EN LOS GCA.

En muy contadas ocasiones se presentan las características y condicionantes de las tareas empleados en los programas contrastados en las investigaciones. Es necesario ajustar y validar las tareas de los programas de aprendizaje a las características de los modelos a investigar ${ }^{(11)}$ y dar difusión a las mismos. En la literatura se considera que en el modelo tradicional las tareas son inespecíficas ${ }^{(2)}$ y definidas, con poco margen para la toma de decisiones del aprendiz ${ }^{(29)}$. Por el contrario, las tareas planificadas bajo el enfoque de la pedagogía no lineal se basan en el aprendizaje situado ${ }^{(18)}$, donde se plantean situaciones de juegos diseñadas bajo diversos condicionantes que plantean un problema que el alumno debe resolver poniendo en práctica sus experiencia previas y su reflexión sobre la práctica ${ }^{(12)}$.
I) LA COMUNICACION DOCENTE - APRENDIZ EN LA TAREA.

La función docente no sólo se centra en el diseño de tareas y sus condicionantes, sino de plantear un proceso de comunicación docente - aprendiz coherente con las teorías que subyacen bajo la pedagogía no lineal y las teorías del constructivismo. La comunicación en el aula es uno de los grandes déficits de los procesos de enseñanza - aprendizaje. Al docente le es más fácil proporcionar información sobre el error y consejos para perfeccionar habilidades para su posterior aplicación. Sin embargo, la presentación de la tarea debe ser coherente con la idea de presentar un problema y mantener una comunicación que favorezca los procesos metacognitivos. El cambio de paradigma está intentar poner en marcha los procesos metacognitivos de los aprendices a través de preguntas para estimular la toma de decisiones y la reflexión.

La dificultad que plantean los docentes es ¿qué preguntas hacemos? y ¿cuándo las hacemos? Es necesario cuestionarse cuáles son las preguntas eficaces en cada situación y ajustarlas al tiempo que disponemos. Para favorecer la adquisición de un conocimiento declarativo y procedimental que de significado a las acciones de juego es necesario que los estudiantes intenten verbalizar lo sucedido y las posibles soluciones a través del análisis, la reflexión y la discusión ${ }^{(20)}$ del ¿qué hacer? ¿cuándo hacerlo? y ¿cómo hacerlo? en cada situación de aprendizaje ${ }^{(33,5)}$.

En numerosos estudios de la literatura científica el control de la comunicación docente aprendiz se supone y no se contempla como una variable que puede condicionar la propia tarea. Los docentes plantean este aspecto como uno de los más complejos para aplicar los modelos comprensivos.

VII) LOS INDICADORES DE LA EFICACIA DE LOS GCA

En cuanto a los aprendizajes de tipo técnico y táctico es necesario incorporar indicadores de rendimiento que sea puedan analizar al jugador en el propio contexto de juego de forma válida y fiable ${ }^{(22)}$. El tipo de pruebas con las que se ha medido el aprendizaje dificulta la comparación de los resultados ${ }^{(23)}$, en ocasiones se desconoce con qué instrumento y cómo se calculan los índices de rendimiento. Hay estudio donde se miden las habilidades técnicas a través de pruebas cerradas y otros utilizan herramientas que permiten analizar la aplicación en el contexto de juego real como el Game Performance Assessment Instrument (GPAI) (26), o el Team Sport Assessment Procedure, (TSAP) ${ }^{(13)} \mathrm{u}$ otras herramientas similares ${ }^{(11,15,34)}$. Las herramientas para la evaluación del alumnado deben ser coherentes con un aprendizaje centrado en juego, los docentes necesitan conocer como emplear estas herramientas y su aplicación en el aula. 


\section{PARA LA APLICACIÓN EN LA EDAD ESCOLAR}

A pesar de las investigaciones en sobre los modelos para la enseñanza del deporte, y de que la literatura otorga numerosas ventajas al enfoque comprensivo se observan dificultades por parte del profesorado para incorporarlo a su práctica docente ${ }^{(5,29)}$. Los docentes manifiestan que tienen dificultades para encontrar materiales dirigidos a los docentes basados en la enseñanza compresiva ${ }^{(5)}$ en un contexto ecológico.

\section{CONCLUSIÓN}

Es necesario realizar investigaciones desde una perspectiva holística e integral. Estudios que controlen en un mismo diseño un mayor número de variables relacionadas con el aprendizaje del deporte, habilidades técnicas, toma de decisiones y conocimiento del juego, así como de variables psico-sociales, como la capacidad de atención y concentración del deportista, la motivación, la satisfacción por la práctica,... para contrastar programas validados por expertos. Además, los investigadores deben tener en cuenta las dificultades del proceso de enseñanza aprendizaje en el contexto escolar y extraescolar, tanto para el aprendiz como para el docente. Es necesario conocer e identificar las dificultades y necesidades formativas que tienen los docentes/entrenadores en ejercicio y fase de pre-servicio para aplicar los modelos de aprendizaje de forma consciente.

La dificultad del cambio de paradigma, hacia una enseñanza basada en la resolución de problemas, es un hecho en la mayoría de aprendizajes escolares. Las investigaciones no pueden ir sólo al qué y cuanto, el futuro está en ir al fondo del asunto, al por qué mejora el aprendiz (neuroaprendizaje) y al cómo aplicar estos modelos.
1. Abad MT, Benito PJ, Giménez FJ, Robles J. (2013). Fundamentos pedagógicos de la enseñanza comprensiva del deporte: Una revisión de la literatura. Cultura. Ciencia y Deporte. 9(23):137-46.

2. Alarcón F, Cárdenas D, Miranda MT, Ureña N, Piñar MI (2010) La metodología de enseñanza en los deportes de equipo. Revista de Investigación en Educación.;:91-103.

3. Araújo D, Travassos B, Torrents C, Vives M (2011) La toma de decisiones en el deporte escolar. Un ejemplo aplicado al fútbol. Innovació en Educació Física $3(2): 1-7$

. Bunker D, Thorpe R (1982). Model for the teaching of games in secondary schools. Bulletin of Physical Education 18(1):5-8

5. Castejon FJ (2015). La investigación en iniciación deportiva válida para el profesorado de educación física en ejercicio [Research on sports initiation valid for physical education teachers in-service]. Retos 28.263-9. . Chow JY Davids $K$ C, Shuttleworth R Renshaw I Araújo D (2007). The Role of Nonlinear Pedagogy in Physical Education. Review of Educational Research.77(3):251-78.

7. Davids K, Bennet S, Button C (2008). The dynamics of skill acquisition. Champaign, IL: Human Kinetics. 8. Den Duyn N (1996). Game sense: Why it makes sense to play games. Sports Coac;19(3):6-9.

9. Den Duyn N (1997). Games Sense. Developing thinking players. Camberra: Australian Sports Commision.

10. García JA, Ruiz LM (2003). Análisis comparatvo de dos modelos de intervención en el aprendizaje del balonmano. Revista de Psicología del Deporte 12(1):55-66.

11. González-Espinosa S, Feu S, García-Rubio J, Antúnez A, García-Santos D (2017). Diferencias en el aprendizaje según el método de enseñanza-apren zaje en el baloncesto. Journal of Sport Psychology 26(Suppl 1):65-70

12. Gréhaigne JF, Caty D, Godbout P (2010). Modelling ball circulation in invasion team sports: a way to promote learning games through understanding. Physica Education and Sport Pedagogy 15(3):257-70.

13. Gréhaigne JF, Godbout P, Bouthier D (1997). Performance assessment in team sports. Journal of Teaching in Physical Education 16:500-16.

14. Griffin L, Mitchell S, Oslin J (1997). Teaching sport concepts and skills: A tactical games approach. Champaign, IL: Human Kinetics.
15. Harvey S, Cushion CJ, Wegis HM, Massa-Gonzalez AN (2010). Teaching games for understanding in American high-school soccer: a quantitative data analysis using the game performance assessment instrument. Physical Education and Sport Pedagogy 15(1):29-54

16. Jones R, Marshall S, Peters D (2010). Can We Play a Game Now? The Intrinsic Benefits of TGfU. European Journal of Ph ysical and Health Education 42(2):57-63.

17. Kirk D, Brooker R, Braiuka S (2003). Enseñanza de los juegos para la comprensión: Perspectiva situada en el aprendizaje de los estudiantes. Ágora para la Educación Física y el Deporte (2-3):154-64.

18. Kirk D, MacPhail A (2002). Teaching games for understanding and situated learning: Rethinking the Bunker-Thorpe model. Journal of Teaching in Physical ducation $21(2): 177-92$

19. Launder AG (2001). Play practice: The games approach to teaching and coaching sports. Champaign: Human Kinetics.

20. López-Ros V, Castejón-Oliva FJ, Bouthier D, Llobet-Martí B (2015). Modelos para una enseñanza comprensiva del deporte. Espacios comunes para el encuentro (y algún desencuentro). Agora para la EF y el Deporte 17(1):45-60.

21. McNeill MC, Fry JM, Wright SC, Tan WK, Tan KS Schempp PG (2004). In the local context: Singaporean challenges to teaching games on practicum Sport, Education and Society 9: 3-32.

22. Memmert D, Harvey S (2010). Identification of non-specific tactical tasks in invasion games Physical Education y Sport Pedagogy 15(3): 287-305.

23. Miller A (2015). Games Centered Approaches in Teaching Children \& Adolescents: Systematic Review of Associated Student Outcomes. Journal of Teaching in Physical Education 34(1):36-58.

24. Mitchell SA (1996). Improving invasion game performance. Journal of Physical Education, Recreation and Dance 67(2):30-3.

25. Nathan S, Haynes J (2013). A move to an innovative games teaching model: Style E Tactical (SET). Asia-Pacific Journal of Health, Sport \& Physics Education. 4(3):287-302.

26. Oslin JL, Mitchell SA, Griffin LL (1998). The game performance assessment instrument (GPAI): Development and preliminary validation. Journal of Teaching in Physical Education 17:231-43. 
skill development, knowledge, and game performan-

Adrieli Martins

$$
\text { Science 12(4):219-36. }
$$

28. Sánchez F (1992). Didáctica de la Educación Física y el deporte. Madrid: Gymnos.

29. Sánchez-Gómez R, Devís J, Navarro-Adelantado V (2014). El modelo Teaching Games for Understanding en el contexto internacional y español: Una perspectiva histórica. Ágora para la Educación Física y el Deporte $16(3): 197-213$

30. Serra-Olivares J, García-Rubio J (2017). La probemáca táctica, clave blemáca táctica clave del diseño representatio de tareas desde el enfoque de la pedagogía no lineal aplicada al deporte. Retos 23(2):261-9.

31. Siedentop D, Tannehill D (2000). Developing teaching skills in physical education 4th ed. Mountain View, CA Mayfield Publishing.

32. Siedentop D. (1998) What is sport education and how does it work? Journal of Physical Education. Recreation \& Dance 69(4):18-20

33. Stolz S, Pill S (2014). Teaching games and sport for understanding: Exploring and reconsidering its relevance in physical education. European Physical Education Review 20(1):36-71.

34. Tallir IB, Lenoir M, Valcke M, Musch E (2007). Do alternative instructional approaches result in different game performance learning outcomes? Authentic assessment in varying game conditions. International Journal of Sport Psychology 38(3):263-82.

35. Tan CWK, Chow JY, Davids K (2012). 'How does TGfU work?': examining the relationship between learning design in TGfU and a nonlinear pedagogy. Physical Education \& Sport Pedagogy 17(4):331-48

\section{RESUMO}

Aline Ferreira ${ }^{1}$

forma de ensino-aprendizagem

do futebol e beisebol

'Instituto Federal de Educação, Ciênci

e Tecnologia do Sul de Minas Gerais

https://doi.org/10.5628/rpcd.17.S1A.59

ALAVRAS CHAVE:

Exergames. Jogos virtuais.

Esportes coletivos.

Este trabalho aborda como os jogos eletrônicos, em especial os Exergames podem contribuir no ensino escolar. 0 objetivo foi avaliar a participação dos alunos utilizando jogos em vivência virtual e real ligadas às modalidades coletivas, e assim, analisar a preferência de ambos nos jogos propostos. Realizado em uma escola municipal de Muzambinho/MG o trabalho direcionou-se principalmente à prática que consistia analisar o desenvolvimento dos alunos. A inclusão dessa ferramenta no ambiente escolar ainda é limitada por seu alto custo, mas temos de lidar com novas necessidades pedagógicas. Sendo assim, os jogos virtuais parecem representar um ambiente favorável para a aprendizagem e participação efetiva desta nova geração de alunos. 